P0001

\title{
IMPROVEMENT OF COLOR APPEARANCE OF URETHANE SKIN SAMPLES BY USING COMPUTER COLOR MATCHING METHOD
}

\author{
Yuki Akizuki et al.
}

DOI 10.25039/x46.2019.PO001

from

CIE x046:2019

Proceedings

of the

29th CIE SESSION

Washington D.C., USA, June 14 - 22, 2019

(DOI 10.25039/x46.2019)

The paper has been presented at the 29th CIE Session, Washington D.C., USA, June 14-22, 2019. It has not been peer-reviewed by CIE.

(C) CIE 2019

All rights reserved. Unless otherwise specified, no part of this publication may be reproduced or utilized in any form or by any means, electronic or mechanical, including photocopying and microfilm, without permission in writing from CIE Central Bureau at the address below. Any mention of organizations or products does not imply endorsement by the CIE.

This paper is made available open access for individual use. However, in all other cases all rights are reserved unless explicit permission is sought from and given by the CIE.

CIE Central Bureau

Babenbergerstrasse 9

A-1010 Vienna

Austria

Tel.: +4317143187

e-mail: ciecb@cie.co.at

www.cie.co.at 


\title{
IMPROVEMENT OF COLOR APPEARANCE OF URETHANE SKIN SAMPLES BY USING COMPUTER COLOR MATCHING METHOD
}

\author{
Akizuki, Y. ${ }^{1}$, Osumi, M. ${ }^{2}$ \\ 1 University of Toyama, Toyama, JAPAN, ${ }^{2}$ Office Color Science, Yokohama, JAPAN \\ akizuki@edu.u-toyama.ac.jp
}

DOI 10.25039/x46.2019.PO001

\begin{abstract}
This paper reports a procedure of making better urethane skin samples which have spectral reflectance factors close to real human skin color data by using a method of computer color matching (CCM). A CCM system based upon absorber and scatter theory by Kubelka Munk and relations equation by Saunderson were used. We prepared a pigments database which accumulated each pigment's spectral reflectance factor and Kubelka-Munk's absorption and scattering coefficient. By using the CCM system and the pigment database, we found the optimal pigment blending ratios and made the new urethane sample's data, which produced the almost same spectral reflectance factors as real skin, significantly better than previous urethane samples in our research.
\end{abstract}

Keywords: Computer Color Matching, Human Skin Color, Spectral Reflectance Factor

\section{Introduction}

Human skin color is controlled by genetic, environmental elements, and health condition. The most important pigments affecting skin color are melanin and hemoglobin concentration. First ${ }_{2}$ melanin determines one's skin color to be darker-or-not by genetic or the amount of ultraviolet radiation. Next hemoglobin concentration determines one's skin color to be reddish-or-not by one's health condition that includes the effects of heat environment condition, amount of exercise, nutrition status, and so on.

Under circulatory failures such as shocked and congested conditions, human skin color changes. The change degree of spectral reflectance factor is greatest with the range from $500 \mathrm{~nm}$ to 600 $\mathrm{nm}$ of Hemoglobin absorption. It is important to identify this change of skin color for emergency medical care like disaster situation.

We had developed database of circulatory dysfunctional skin color taking consideration of age and gender by using Japanese healthy subjects' skins which are artificially shocked or congested in part of upper arms (AKIZUKI 2016). The urethane skin model samples; "previous urethane samples" named in this paper, which had similar spectral reflectance factors as real skin under circulatory dysfunction had been developed in previous paper (AKIZUKI 2017). In consequence, the spectral reflectance factor curves of previous urethane samples were closer to real skin than the commercially available skin color paper charts "paper charts" at longer than $580 \mathrm{~nm}$, but they still did not match sufficiently.

This paper reports a procedure of making better urethane skin samples which have spectral reflectance factors close to real human skin color data by using a method of computer color matching (CCM).

\section{Procedure}

\subsection{Computer Color Matching System}

In paint technology, CCM is commonly used for development of paint application. A CCM system created by the second author (OSUMI, 2014) was used in this study. This CCM system concerns the behavior of lights transmitting through painting film with scattering characteristics, based upon absorber and scatter theory by Kubelka and Munk and relations equation by Saunderson. Figure 1 shows the major functions of this CCM system. 
$\mathrm{K}$ is the absorption coefficient of Kubelka-Munk, and $\mathrm{S}$ is the scattering coefficient of that. $\mathrm{R}_{\mathrm{W}}$ and $R_{B}$ are the reflectance of two parts of a basement material in this research. We used BYKcharts as the basement materials which were test charts for measuring color and opacity of painting materials such as pigments, which conform to international standards ASTM-D344 and ISO6504-3. The reflectance of white part of the BYK-chart is $89.4 \%$, and the reflectance of black part of BYK-chart is $6.3 \%$. Rwc is the reflectance of a painting film (a painted part by colored urethane in this study) on the white part of BYK-chart, and $R_{B C}$ is the reflectance on the black part of BYK-chart. $X$ is the painting film thick $(200 \mu \mathrm{m}$ in this study).

$$
\begin{aligned}
& A=R_{W C} \cdot R_{B}-R_{B C} \cdot R_{W} \\
& B=\left\{R_{W}-R_{B}\right\}\left\{1+R_{W C} \cdot R_{B C}\right\}-\left\{R_{W C}-R_{B C}\right\}\left\{1+R_{W} \cdot R_{B}\right\} \\
& R_{\infty}=\left\{-B+S q r t\left(B^{2}-4 A^{2}\right)\right\} / 2 A \\
& S=\ln \left[\left\{\left(R_{\infty}-R_{W}\right)\left(1 / R_{\infty}-R_{W C}\right)\right\} /\left\{\left(R_{\infty}-R_{W C}\right)\left(1 / R_{\infty}-R_{W}\right)\right\}\right] /\left\{X \cdot\left(1 / R_{\infty}-R_{\infty}\right)\right\} \\
& K=S\left(1-R_{\infty}\right)^{2} /\left(2 R_{\infty}\right)
\end{aligned}
$$

Figure 1 - Major Functions at CCM System of this research (Osumi, 2014)

\subsection{Pigment Database}

Before calculation by this CCM system, we prepared a pigments database which accumulated each pigment's spectral reflectance factor, it's Kubelka-Munk's absorption coefficient (K) and it's scattering coefficient $(\mathrm{S})$.

A Transparent urethane (made by Takebayashi Chemical Industry Co.) for painting on BYKchart was clear and colorless, and comprised $50 \%$ of polyol and $50 \%$ of hardener polyisocyanate. We used 12 pigments made by Mikuni-Color Ltd.; 8 pigments of ZA series for urethane resin paint (White 2000, Red T-220, Yellow 2300, Yellow 5000, Green T-219, Blue 2200, Brown 6600 and Black 2900), and 3 colloidal pigments of MHI series (Red \#310, Magenta \#889, Yellow \#340 and Yellow \#593).

A certain pigment or blended pigment (mixed $30 \%$ of the pigment and $70 \%$ of "ZA White 2000 ") was added to $100 \mathrm{~g}$ transparent urethane with density of $1 \%, 3 \%$, and $5 \%$. And then the colored urethane was painted on BYK-chart with $200 \mu \mathrm{m}$ thick. After the colored urethane on BYK-chart was dry completely, we measured the spectral reflectance factors of two painted parts on white/black part of BYK-chart by a spectrophotometric colorimeter. The colorimeter was CM$2600 \mathrm{~d}$ made by Konica-Minolta, and set measuring conditions as specular component excluded, 10 degrees visual field, $3 \mathrm{~mm}$ in diameter, and standard illuminant D65 for calculation values of CIE L*a*b*. The six data of a certain pigment were entered into this CCM system to repetitively calculate Kubelka-Munk's absorption coefficient $(K)$ and its scattering coefficient (S). This pigment database was created by operating these procedures for all 12 pigments.

\subsection{Preparation for Blending Pigment}

A certain pigment blending ratio of a certain reference spectral reflectance factor was calculated by using the CCM system and the pigment database.

We used the Kubelka-Munk's absorption (K) and it's scattering coefficient (S) from this pigment database, Kubelka's absolute two-constancy equation, and Duncan's color mixing equation for the calculation by this CCM system. The calculation by this CCM system was performed to achieve two goals. The first goal is that the color difference $\Delta E^{*} a b$ of $C I E L^{*} a^{*} b^{*}$ between $a$ target spectral reflectance factor and a reference one (real skin color's reflectance data in this study) to be the 0 value. The second goal is the error sum of squares of spectral reflectance factors between the target and the reference to reach the minimum value. 
The calculation condition was $5 \%$ density of blended pigments against $100 \mathrm{~g}$ transparent urethane. And we got the optimal pigment blending ratio to produce the target reflectance factor closer to the reference spectral reflectance factors.

\section{Necessary Pigments for Producing Human Skin Color}

In our previous paper (AKIZUKI, 2016), we categorized the real skin color results by three circulatory states (healthy, shocked and congested) and four subject groups based on age and gender. And twelve spectral reflectance factors of typical human skin were defined as the reference spectral reflectance factors in this study.

By using the CCM system and the pigment database, we found the optimal pigment blending ratios and made the new urethane sample's data. Figure 2 shows the eight necessary pigments' results of spectral reflectance factors on white/black part of BYK-chart.
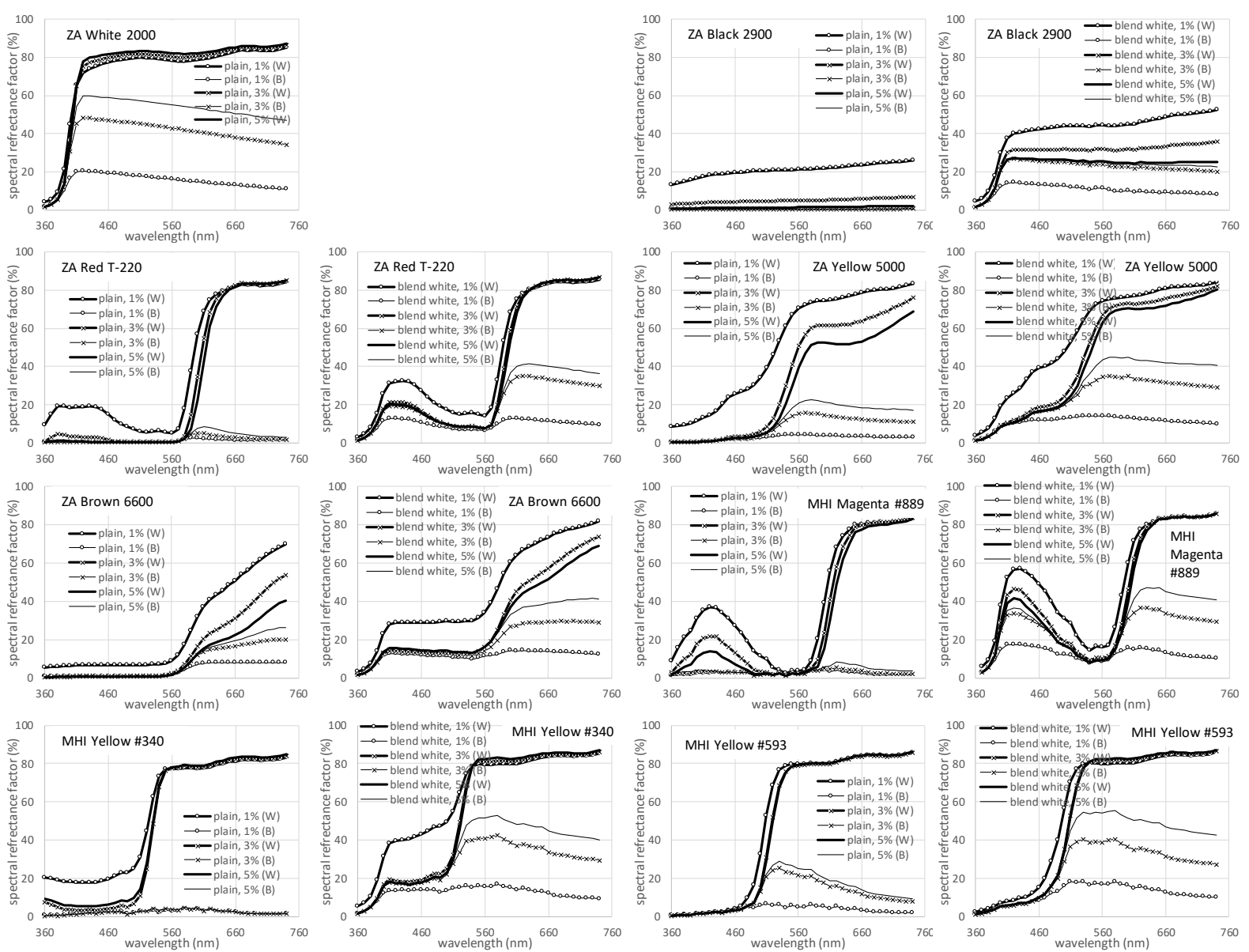

Figure 2 - Spectral Reflectance Factors of Necessary Pigments for Producing Human Skin Color (three density results on white(W)/black(B) part of BYK-chart)

\section{Comparison with Real Human Skin Data}

\subsection{Spectral Reflectance Factor}

The spectral reflectance factors of real human skin data, paper charts (AKIZUKI, 2016), previous urethane samples (AKIZUKI, 2017), CCM simulation data, and new urethane samples' data on white/black part of BYK-chart which produced based on the optimal pigment blending ratio are shown in Figure 3. Each new urethane sample's data was made from the six chosen pigments among the eight pigments in Figure 2.

As compared with the paper charts, the spectral reflectance factors of the previous urethane samples were closer to the real human skin data. But within the range from $500 \mathrm{~nm}$ to $600 \mathrm{~nm}$ 
which is the characteristic of hemoglobin, the gap of spectral reflectance factors was significant. On the other hand, the spectral reflectance factors of the new urethane samples' data were close to real human skin data. Especially many results of the new urethane samples' data on white part of BYK-chart were fit with real human skin data.
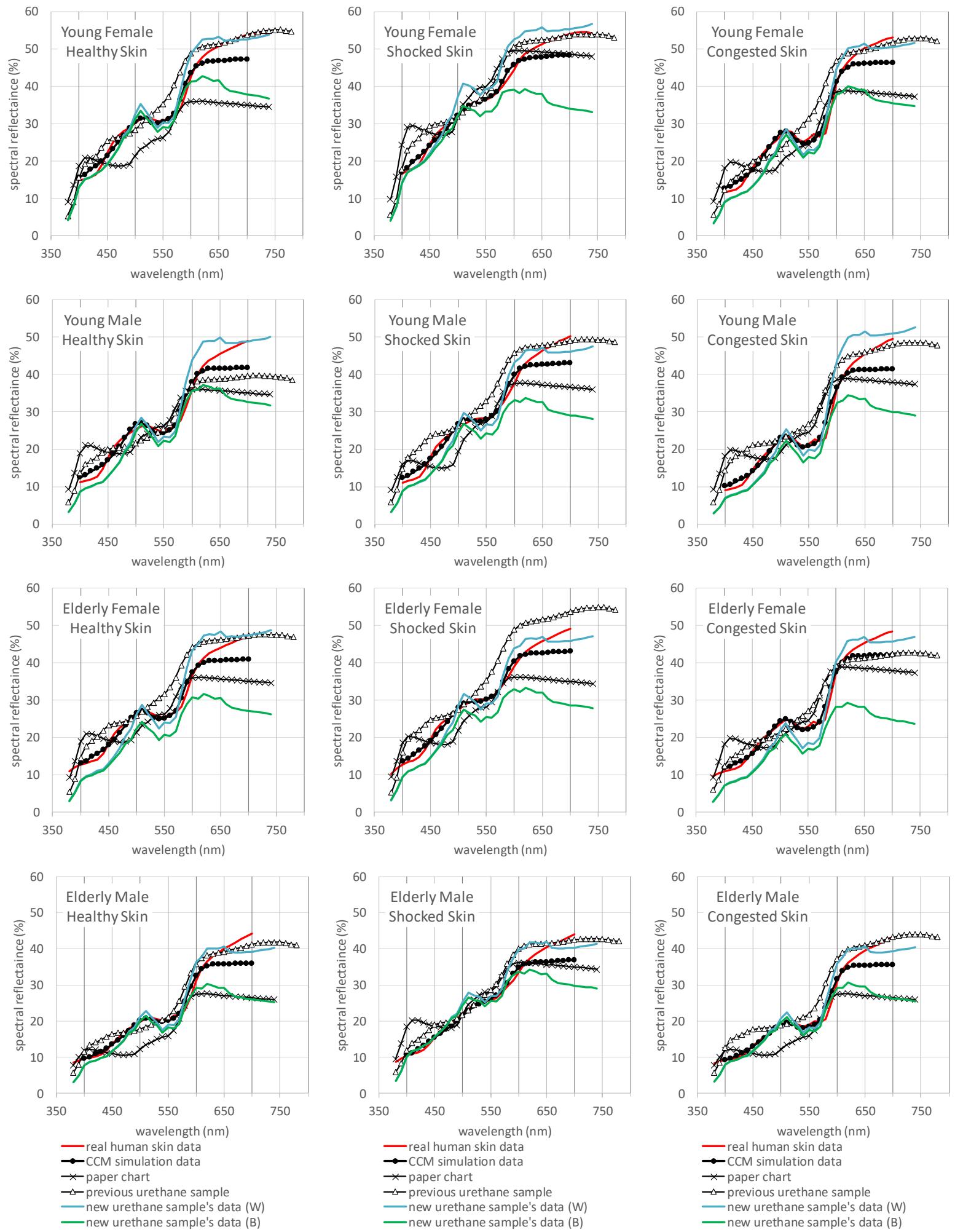

Figure 3 - Spectral Reflectance Factors of Real Human Skin and Skin Samples with CCM simulation data (three circulatory states and four subject groups) 


\subsection{Error Sum of Squares of Spectral Reflectance Factors}

In Figure 3, the spectral reflectance factor of CCM simulation data was greater than the real human skin data more than $600 \mathrm{~nm}$ and made significant gap. However otherwise (less than $600 \mathrm{~nm}$ ) no gap was found. Based on the results, at first, we calculated error sum of squares of spectral reflectance factors between the paper charts or the previous urethane samples or the new urethane samples' data and reference real human skin data. And then, the ratios of the error sum of squares of skin samples (the paper charts, the previous urethane samples, and the new urethane samples' data) to CCM simulation data were calculated. The results are shown in Table 1. The bold number in Table 1 means the nearest sample to CCM simulation data in the comparison.

Within the range from 400 to $700 \mathrm{~nm}$, many results of the new urethane samples' data on white part of BYK-chart were the nearest. Within the range from $500 \mathrm{~nm}$ to $600 \mathrm{~nm}$ where the real human skin color data and CCM simulation data fit well, either of new urethane sample's data on white/black part of BYK-chart were the nearest ones in most cases. Therefore, the new urethane sample's data were considered to be able to reproduce real human skin.

Table - 1 Ratio of Error Sum of Skin Samples to CCM simulation data

\begin{tabular}{|c|c|c|c|c|c|c|c|c|c|c|c|c|c|}
\hline & \multicolumn{3}{|c|}{ Young Female } & \multicolumn{3}{|c|}{ Young Male } & \multicolumn{3}{|c|}{ Elderly Female } & \multicolumn{3}{|c|}{ Elderly Male } \\
\hline & & $\mathrm{H}$ & $\mathrm{S}$ & $\mathrm{C}$ & $\mathrm{H}$ & $\mathrm{S}$ & $\mathrm{C}$ & $\mathrm{H}$ & $\mathrm{S}$ & $\mathrm{C}$ & $\mathrm{H}$ & $\mathrm{S}$ & $\mathrm{C}$ \\
\hline \multirow{4}{*}{ 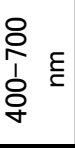 } & paper chart & 13.65 & 5.03 & 7.97 & 5.85 & 5.62 & 4.42 & 6.28 & 9.19 & 5.54 & 8.00 & 3.41 & 7.72 \\
\hline & previous urethane sample & 2.23 & 2.39 & & 2.69 & & & 3.97 & 6.67 & & & & .01 \\
\hline & new urethane sample's data (W) & 1.11 & 3.17 & 1.15 & 1.68 & 1.17 & 1.75 & 2.41 & 1.65 & 1.98 & 0.72 & 1.06 & 0.71 \\
\hline & new urethane sample's data (B) & 5.89 & 14.95 & 7.70 & 5.64 & & 6.97 & 15.18 & 16.95 & 20.88 & 6.02 & 4. & 5.65 \\
\hline \multirow{4}{*}{ 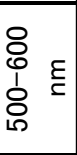 } & \begin{tabular}{|l|} 
paper chart \\
\end{tabular} & 18.33 & 26.38 & 7.11 & 4.68 & 5.90 & 12.22 & 6.69 & 9.70 & 10.48 & 15.74 & 13.16 & 8.48 \\
\hline & previous urethane sample & 20.04 & 16.69 & 16.73 & 4.19 & 22.20 & 17.71 & 32.33 & 52.42 & 11.45 & 8.23 & 24.24 & 13.37 \\
\hline & new urethane sample's data (W) & 7.31 & 37.55 & 3.00 & 6.18 & 6.18 & 5.75 & 8.46 & 9.08 & 5.91 & 5.04 & & 4.48 \\
\hline & new urethane sample's data (B) & 1.90 & 11.74 & 3.04 & 2.38 & 9.98 & 3.77 & 15.07 & 25.08 & 15.34 & 2.71 & 3.56 & 1.44 \\
\hline
\end{tabular}

\section{Conclusions}

By using the CCM system and the pigment database, we found the optimal pigment blending ratios and made the new urethane sample's data, which produced the almost same spectral reflectance factors as real skin, significantly better than previous urethane samples.

However, there are some parts that do not match well enough with CCM simulation data. Therefore, it is necessary to improve the skill of blending pigments.

We will conduct further study to develop new skin urethane samples which has consideration for real skins' transmittance. Ultimately, we aim at the development of light source which is effective in distinguishing skin color changes for medical care

\section{Acknowledgments}

This research was supported by the Japan Society for the Promotion of Science (JSPS) KAKENHI Grant Number JP16K06607 (Program Leader: Yuki Akizuki). The authors would like to thank Mr. Yuki Yamamoto of Mikuni-Color Ltd for their cooperation in making the pigments' database for this CCM simulation.

\section{References}

AKIZUKI, Y. and OHNO, Y. 2016. Study on Spectral Characteristics for Identification of Skin Colour of Injured Japanese Persons at Disaster Sites, Proceedings of the 4th CIE Expert Symposium on Colour and Visual Appearance x0430, 179-189.

AKIZUKI, Y. and OHNO, Y. 2017. Preliminary Study on Spectral Characteristics for Identification of Skin Colour under Circulatory Dysfunction using Artificial Skin Samples, Proceedings of the CIE Midterm Meeting x044, 303-313.

OSUMI, M. 2014. The Human Skin Colorimetric and Evaluation Way applied Spectral Imaging and Analysis of Optics Structure, Proceedings of AIC Interim Meeting, 660-669. 